\title{
Investigating the Usability of PDAs with Ageing Users
}

\author{
Sheila Mc Carthy \\ University of Ulster at Magee \\ Northland Road, Derry BT48 \\ $7 \mathrm{JL}$ \\ Northern Ireland, UK \\ +44 (0)2871375157 \\ McCarthy-S2@ulster.ac.uk
}

\author{
Heather Sayers \\ University of Ulster at Magee \\ Northland Road, Derry BT48 \\ $7 \mathrm{JL}$ \\ Northern Ireland, UK \\ +44 (0)2871375148 \\ hm.sayers@ ulster.ac.uk
}

\author{
Paul mc Kevitt \\ University of Ulster at Magee \\ Northland Road, Derry BT48 \\ 7JL \\ Northern Ireland, UK \\ +44 (0)2871375433 \\ p.mckevitt@ulster.ac.uk
}

\begin{abstract}
Mobile technologies have the potential to enhance the lives of ageing users, especially those who experience a decline in cognitive abilities. However, diminutive devices often perplex the aged and many HCI problems exist. This research ultimately aims to develop a mobile reminiscent application for ageing users entitled MemoryLane. This application will use artificial intelligent techniques to compose and convey excerpts from a lifetime's memories to the user in a multimodal storytelling format. The proposed deployment platform for MemoryLane is a Personal Digital Assistant (PDA). The initial stage of this research, a HCI pilot study was recently conducted with a sample of ageing users, the study aimed to investigate the usability of a PDA. This paper documents the methodologies employed in this pilot study and its subsequent results. The next stages of the research are also identified and discussed.
\end{abstract}

\section{Categories and Subject Descriptors}

K.4.2. [Social Issues]: Assistive technologies for persons with disabilities.

\section{General Terms}

Design, Human Factors.

\section{Keywords}

PDA, usability, HCI, elderly, cognitive decline, memory loss, gerontechnology, storytelling, MemoryLane.

\section{INTRODUCTION}

The ageing population is dramatically increasing, especially in the more economically developed countries of the world. According to the 2001 census the UK now has more people aged over 60 than under 16 years. 1.1 million people are now aged over 85, and by 2050 the number of centenarians is expected to have increased eighteen times [1]. Cognitive decline is part of the natural ageing process affecting individuals at varying rates, and catering for such a diverse

\footnotetext{
(C) Sheila Mc Carthy, Heather Sayers \& Paul Mc Kevitt, 2007 Published by the British Computer Society Volume 2 Proceedings of the 21st BCS HCI Group Conference

HCI 2007, 3-7 September 2007, Lancaster University, UK Devina Ramduny-Ellis \& Dorothy Rachovides (Editors)
}

sector requires detailed analysis. Mobile computing is commonplace and offers the potential to be harnessed as a tool to assist many of these ageing people. However, many usability problems exist and this potential is very often not maximised. Mobile technologies can assist ageing users to live independently and maintain a high quality of life, in turn minimising the emotional and financial strain often caused by nursing home accommodation. The usability problems identified in this research will contribute to the development of a set of design guidelines which will aim to assist in the design of PDA based applications for ageing users. This research is underpinned by the larger research areas of gerontechnology and HCI usability studies.

\subsection{Gerontechnology and HCI}

Due to the increasing numbers of the ageing population, they have become the focus of much research designed to improve, prolong and enhance their lives. Gerontology is the study of ageing people and of the social, psychological and biological aspects of the ageing process itself, as distinct from the term Geriatrics, the study of the diseases which afflict ageing people. Gerontechnology, the merger between gerontology and technology is a newer genus, concerning itself with the utilisation of technological advancements to improve the health, mobility, communication, leisure and environment of ageing people. Therefore gerontechnology is heavily concerned with the ways in which ageing people interact with computers and technology. In a recent paper Zajicek [8] reflects upon established HCI research processes and identifies certain areas in which this type of research differs significantly from other research disciplines.

\subsection{HCI Usability Studies}

Myriad HCI usability studies are being conducted in the area of computers and ageing users, but substantially less are being conducted into the specifics of how ageing people interact with mobile devices, despite the fact that active researchers within this area have discussed the benefits of mobile devices to ageing people, and have highlighted the need to learn more to support designing for this genre [3]. An initial PDA usability study conducted by Siek et al. [6] compared differences in the interaction patterns of ageing users and younger users. This work attempted to ascertain whether ageing people, who may be subject to reduced cognitive abilities, could effectively use PDAs. This initial research was conducted with a small sample of 20 users, a control group of 10 younger users aged 25-35, and 10 older users aged 75-85 years and was restricted to the monitored analysis of the participants' abilities to perform 5 controlled interactive tests using a Palm Tungsten T3 PDA. The findings of this study failed to identify any major differences in 
the performance of the two groups possibly due to the fact that the ageing users group were allowed extra practice time privileges. Siek et al.'s work offered an early insight into the nature of the field work for this research.

\section{METHODOLOGY AND DESIGN}

The aim of this research is to develop a user-friendly PDA application called MemoryLane to assist ageing users recall past life events and memories as they experience the natural cognitive declines associated with the ageing process. The preferred deployment platform, a DELL Axim X51v PDA device is pictured beside an impression of the proposed MemoryLane prototype in Figure 1. The methodology adopted in this research is the User Sensitive Inclusive Design (USID) approach proposed by Newell and Gregor which focuses on universal usability. This new methodology extends User Centred Design by developing technological systems for everyone, to include those with disabilities and mutatis mutandis for other minority groups [5]. Close attention will also be paid to the findings that mutual inspiration is the most effective approach in developing new technology for ageing people [2] and the recent study by Zajicek [8] into the various aspects of HCI for ageing people. MemoryLane will be designed to support multimodal input via a touch screen and possible use of simple voice control commands. The benefits of multimodal interaction are widely acclaimed and the design of MemoryLane will assure a multimodal interface which will accommodate ageing users with different capabilities, expertise or expectations [4]. MemoryLane will also provide multimodal output in the form of images, video, audio and text to speech synthesis. It is thought that MemoryLane will incorporate a hybrid artificial intelligent system to compose life-caching data into appropriate and pleasing 'stories' for the user.

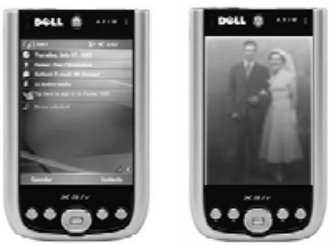

Figure 1: Dell Axim X51v PDA and an Impression of the Proposed MemoryLane Prototype

\section{PILOT STUDY}

The initial stage of this research began with a preliminary HCI pilot study conducted with a sample of older users and aimed at investigating the usability of a PDA. Prior to conducting interviews many preliminary visits were initially required to gain trust and build a rapport with the ageing participants. Silverman [7] recommends that when interacting with a sample, researchers should note all they see and hear and also how they behave and feel they are treated.

\subsection{Sample Selection}

The pilot study sample comprised 15 participants of apparent good health. The sample was aged between 55 and 82 years and included 6 males and 9 females. Participants were selected from four different sources, 6 attended an Age Concern centre, 3 were members of The University of the 3rd Age, 2 were day patients of a local Nursing Unit and the remaining 4 were selected at random from responses received from volunteers.

\subsubsection{Personal Backgrounds}

Of the 15 participants 14 were right-handed. No participants were colour-blind and 12 wore glasses. 5 admitted to experiencing minor vision problems when viewing a TV or computer screen and 3 suffered from slight hearing loss. All but one participant lived in their own home and 13 agreed that they had more free time now than they used to have. 7 of the 15 reported that they had more disposable income than 10 years ago, 6 thought they had less. Most participants watched TV on a daily basis, the favoured programs ranging between soap operas news and weather, sports and documentaries. No one expressed a preference for technical or computer programs. 11 participants read on a daily basis, but none expressed a preference for reading any form of technical or computing literature.

\subsection{Interviews \& Questionnaires}

Each participant was interviewed separately in a one-to-one structured interview format in familiar surroundings. The interviews involved completion of a detailed questionnaire, a demonstration of how to interact with a PDA by the researcher, followed by observation of participants' capability in attempting to complete pre-set interactive PDA tasks.

Initial research for the questionnaire design discovered that questions requiring prose type answers took participants too long to complete, during which they often became frustrated and seemed to prefer yes/no or tick box answers. Prose answers also proved ambiguous and often difficult to quantify, therefore the questionnaire followed the 5 point Likert- type scale giving participants 5 optional answers. The ensuing questionnaire was divided into sections A and B. Section A of the questionnaire was designed to acquire background information regarding participants' physical characteristics, socio-economic factors, perceived technical abilities, prior exposure to technology and personal opinions of modern day technology. Section B of the questionnaire was designed to be completed in conjunction with undertaking the interactive PDA tasks; this section determined the participant's ability to complete the set tasks and ascertained their HCI preferences. This section centered on questions regarding preferred interaction modalities and aspects and elements of the PDA hardware and software. As part of section $\mathrm{B}$, participants were asked to attempt 6 basic tasks on the PDA as illustrated in Figure 2. This section of the interview was videotaped where possible, in conjunction with the participant's approval.

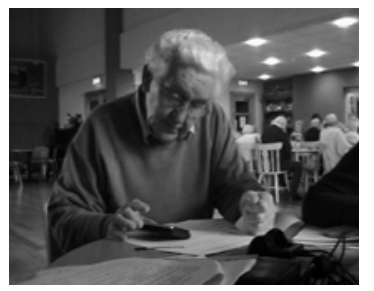

Figure 2: Participant Interacting with PDA

\section{RESULTS}

The following sub sections document the findings of the pilot study and are organised into sections which reflect the format of the questionnaire.

\subsection{Technical Experience}

Section A of the questionnaire details the levels of prior technical exposure and experience encountered by the 15 participants. Questions focused on participants' usage of household technical devices, with a view to investigating if those participants with increased exposure to technical devices found PDA interaction easier than their counterparts. 
Phones were the first device under scrutiny, 8 participants had a regular corded home telephone, 6 owned regular cordless telephones and 9 used a mobile phone. When asked, no one rated their ability to use a mobile phone as 'excellent', 5 considered their ability as 'good' and 7 considered themselves as 'poor' phone users. All participants owned a TV, but only 4 had ever used Teletext. 8 participants owned both a VCR and DVD player and only 2 of the participants said that they did not use a remote control to operate these devices. This demonstrated that the majority of the participants were familiar with operating buttons on a basic hand-held device such as a remote control. The majority of participants said that they did not require any assistance with programming or operating household devices such as timers, alarms, radios and microwaves, however not all actually owned programmable devices. 4 said that they found such devices complex and did require assistance.

As expected, the findings of the pilot study concluded that those participants who owned and used a mobile phone were generally more predisposed to engaging with the PDA, as were those who extensively utilised their household appliances. Those participants who declared minimal usage of telephones, remotes and household devices tended to show more apprehension toward the PDA.

The main reason for computer use among this age bracket was for email purposes and the majority of users proclaimed to be self-taught. Only 5 participants owned their own computer and 3 used a computer on a daily basis. 9 said they had never used a computer; however 10 reported that they had access to a computer if desired. Only 1 participant had prior knowledge of a PDA, the remainder had never heard of them. Just half the participants said they were interested in learning about new technologies, and less than this thought it important for them to keep abreast with technological advances.

\subsection{Computing Expertise}

It was hypothesised that participants who regularly socialised with younger people (in the age bracket of 25 to 55) would have a higher exposure to modern day technologies than those who do not regularly socialise with people of this age, and would rate their own level of computer knowledge as significantly higher. However this generalisation proved not to be the case, as can be seen in Figure 3.

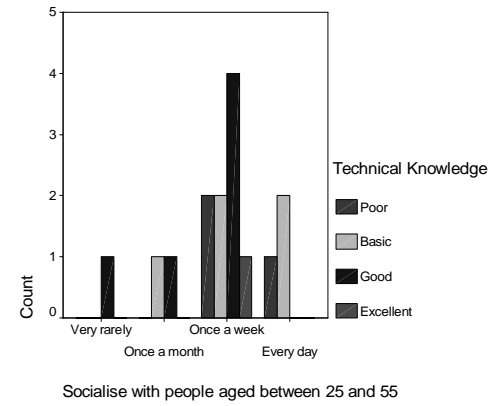

Figure 3: Technical Knowledge \& Social Interaction

Participants who had the highest social interaction with younger people (on a daily basis) described their knowledge levels as merely poor and basic. Participants who socialised weekly with younger people were divided over rating their computing ability. 4 did describe their computing knowledge as good and 1 as excellent; however, 4 from this group also rated their knowledge as poor and basic. Participants who socialised on a monthly basis with 25 to 55 year olds described their knowledge levels as basic and good. The remaining participants, who very rarely socialised with younger people, actually described their knowledge levels as good.

Participants' perceptions of their own expertise and knowledge was occasionally subjective, some rated themselves highly and later professed to having never used a computer or a mobile phone, while others, who often used computers, criticised their abilities and rated their knowledge as inferior; one even producing a mobile phone from his pocket to demonstrate how poorly he believed he used it. However, despite the differing impressions participants held of themselves, the findings indicate that in contrast to the hypothesis, regular contact between older and younger people does not seem to generate noteworthy increased knowledge of modern day technologies in the elderly.

It was further hypothesised that participants' previous occupations would have direct bearing on their perceived computing ability, where those with an academic background would display a heightened computing ability. However, as displayed in Figure 4, this again was not the case.

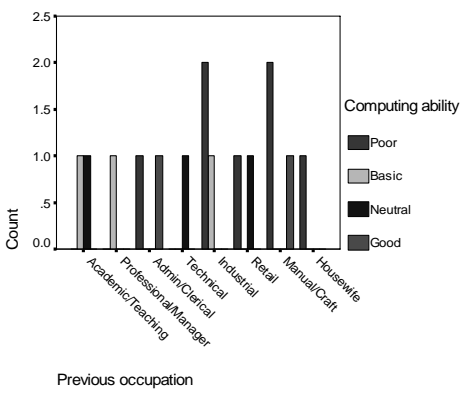

Figure 4: Background \& Computing Ability

None of the participants, irrespective of previous occupation, rated their computing ability as 'excellent'. Participants with academic and managerial backgrounds rated their expertise as merely 'basic', with the highest ratings appearing from those with clerical and manual backgrounds. The poorer self-ratings came from those with clerical, industrial and manual backgrounds. Again these findings did not hold true with the hypothesis and were subject to participants' differing perceptions of their own prowess. These findings would seem to illustrate that there is no definite correlation between the previous occupations of this age group and their perceived computing abilities. Overall, due to the subjectivity of the participants and their lack of benchmarks for comparison, is awkward to quantify the competent levels of computer literacy among the sample. It would seem that the participants too had difficulty in accurately rating their own computing and technical abilities, a conundrum which may account for apprehension in embracing technology.

\subsection{Interactive Tasks}

Task 1 involved navigating a menu structure and locating 3 files containing text of various colours, features and sizes. The first file contained 15 lines of text written in different colours and participants were requested to select their preferred 5 text colours. The second file contained 6 lines of text written in various text styles and the participants were asked to rate their preferred 3. The third file contained 5 lines of text written in different text sizes, 8 to 16 and the participants were asked to select only one preferred text size. The participants had difficulty navigating the menu structure unaided; however the study found that the participants' preferred text colours were: black, red, navy, green and blue. They also preferred text in 
'Normal' format, followed by embolden text and lastly underlined text and the preferred text size among participants was size 10 font. During this task 12 of the 15 were eventually able to use the on-screen scroll bar when aided. Task 2 required participants to locate and view 3 media files. The first was a photograph of the researcher, the second an audio clip of waltz music and the third a video file of line dancing. Participants were then asked to comment on how easily they could view and hear these files. Again, the participants had difficulty when attempting to navigate the menu structure and find and open the required media files. However, when found, all participants were able to see and hear the associated content.

Task 3 involved a dual interaction with a bespoke application called 'Button Press Task'. This task required participants to navigate through a 12 on screen buttons by firstly using the 5way navigational button and then repeat the procedure using the stylus. Participants were asked to indicate their preferred mode of interaction. This task proved one of the more difficult for the participants, the majority found the 5-way navigational button extremely complex and small to use, and all preferred using the stylus (or their finger). Tasks 4 and 5 centred on text entry. Firstly participants were asked to enter their name via the onscreen keyboard, and then subsequently write their name using a free-hand format. The participants were asked to identify which method they preferred. The participants also experienced significant difficulty with this task and required considerable assistance, and not all participants were able to fully accomplish the task, of those who did the majority preferred the 'neatness' of the keyboard option to the 'vagueness' of the free-hand entry.

The final Task 6 instructed each participant to record and replay a simple voice message. Again, no participants were able to accomplish this task on their own, but when aided, participants were able to record and replay their own voice message on the PDA. For completeness the PDA's program buttons were also discussed as were the participants' preferences for screen orientation between portrait and landscape, where the majority preferred the PDA screen in portrait orientation. All participants found the menu structure difficult to navigate, and most found the PDA screen size easy to view but were evenly split over their preference for the interface usage of an icon or text. All participants were able to grip and hold the stylus. No one reported that the PDA felt heavy to carry, or awkward to hold.

\section{CONCLUSIONS}

It was clear from the outset that the participants found the PDA extremely complicated to use and had difficulty even knowing where to start; no one found the interface instinctive or intuitive. This was evidenced by the level of assistance requested and given. Despite the functionality of a PDA being demonstrated beforehand, not one of the participants could carry out even the most basic of tasks unaided. There was also a noticeable level of general disinterest in applications hosted on the PDA; none were of particular personal appeal to the participants. For example most thought that its functions as a calendar or diary were of little interest as they preferred a pen and diary. When asked, many agreed that they would certainly be more interested, and inclined to engage with the PDA if it provided an application of personal interest, such as MemoryLane. However, despite participants initially expressing concern about being unable to partake in the study due to their lack of computer knowledge, and the difficulties incurred during the tasks, many participants said they actually enjoyed the experience of PDA interaction. Most felt that their skills would improve if they had more time with the PDA and some expressed a desire to learn more about a PDA given the desired surroundings and instructor. The portability of a PDA appealed to the majority of participants who remarked on it being 'small enough' to fit into a handbag or breast pocket. This would imply that many elderly users possess a genuine interest in engaging with mobile technologies and that a PDA has a certain appeal to many ageing, however, due to complex interfaces many choose not to experiment with such devices. These findings suggest that the interface for MemoryLane must strive to be simplistic, usable and intuitive to be successfully deployed on a PDA.

\section{FUTURE WORK}

This pilot study has paved the way for the next step in this research, a larger HCI requirements analysis will investigate how elderly people reminisce and recall past memories, this study will lead to the design and implementation of MemoryLane.

\section{ACKNOWLEDGMENTS}

The authors would like to express gratitude to Prof Mike McTear and Dr. Norman Alm for their input and to Dr. Kevin Curran and Professors Bryan Scotney and Sally McClean for their valuable advice and guidance. The authors would also like to extend appreciation to the pilot study participants who took the time to contribute to the research.

\section{REFERENCES}

[1] Directgov (2006), National Statistics, Census 2001, http://www.statistics.gov.uk/census/

[2] Eisma, R., Dickinson, A., Goodman, J., Mival, O., Syme, A. \& Tiwari, L. (2003), Mutual Inspiration in the Development of New Technology for Ageing users, Include 2003, 252 - 259.

[3] Goodman, J., Brewster, S. \& Gray, P. (2004), Older People, Mobile Devices and Navigation, HCI and the Older Population. Workshop at the British HCI 2004, Leeds, UK, Sep 2004, 13 - 14.

[4] Lazar, J., (2007), Universal Usability; Designing Computer Interfaces for Diverse Users, John Wiley \& Sons Ltd., The Atrium, Southern Gate, Chichester, West Sussex, England

[5] Newell, A.F. \& Gregor, P. (2000), User Sensitive Inclusive Design, ACM Conference on Universal Usability 2000, ACM Press, New York, USA, 39 - 44.

[6] Siek, K.A., Rogers, Y. \& Connelly, K.H. (2005), Fat Finger Worries: How Older and Younger Users Physically Interact with PDAs, INTERACT 2005, eds. M.F. Costabile \& F. Paterno, Springer Berlin, 267 - 280.

[7] Silverman, D., (2000), Doing Qualitative Research; A Practical Handbook, SAGE Publications Ltd, 6 Bonhill Street, London.

[8] Zajicek, M. (2006), Aspects of HCI research for elderly people, Universal Access in the Information Society, vol. Volume 5, Number 3, 279 - 286. 